\title{
Design and Control of a Robotic Assistive Device for Hand Rehabilitation (RAD-HR)
}

\author{
Christopher Spiewak, Mohammad Arifur Rahaman², Mohammad Habibur Rahman', Roshan D'Souza', \\ Maarouf Saad $^{3}$ \\ ${ }^{1}$ Biorobotics Lab, University of Wisconsin-Milwaukee \\ 3200 N. Cramer Street, Milwaukee, WI, USA \\ ${ }^{2}$ Mechatronics (Man \& Machine) lab, Khulna University of Engineering \& Technology, Bangladesh \\ ${ }^{3}$ École de technologie supérieure, Montreal, QC, Canada \\ cjspiewak@uwm.edu; rahaman.arifur02@gmail.com; rahmanmh@uwm.edu; dsouza@uwm.edu, maarouf.saad@etsmtl.ca
}

\begin{abstract}
To assist the elderly and/or physically disabled people who no longer possess proper function of their hand motion, we have been developing a robotic assistive device to rehabilitate the hand motions to improve quality of life. The proposed assistive device will be comprised of three degrees of freedom enabling basic movements and hand function. In this research we have focused on the modeling and control of the proposed assistive device. To achieve the dynamic simulation of the developed model, nonlinear computed torque control technique was employed. In the simulation, the trajectory tracking performance of the controller was evaluated.
\end{abstract}

Keywords: Biorobotics, Rehabilitation, Nonlinear Control, Robotic Assistive Device, Exoskeleton

\section{Introduction}

There are an estimated 6.8 million Americans 20 years old, and older who have had a stroke [1]. Many are affected with paralysis, and/or spasticity after a stroke. Paralysis is the inability of a muscle to move properly. Spasticity is a where muscles are stiff, and resist being stretched. Hands are vitally important for numerous functions as part of our daily lives. The rehabilitation of hand motion with the application of a robotic assistance devices sees significant improvements in motor impairment, and functionality recovery of the upper-limb after therapy [2]. To assist disabled individuals with impaired upper extremities we have been designing wearable robots to provide rehabilitation therapy [3] to the upper extremity. The purpose of this research is to design a robotic assistive device (RAD-HR) for the rehabilitation of impaired hand motion. Focusing on immediate post-injury recovery, and rehabilitation. Which allows for the simplification of design to a robotic exoskeletal glove. Simplification of design allows for the recovery, and rehabilitation of basic hand motion after stroke or various injuries.

Compared with conventional treatment, robotics assisted rehabilitation have great advantages in terms of clinical, and biomechanical measures. Robotics assisted rehabilitation have a larger improvements in the Fugl-Meyer assessment of motor impairment [4]. The Fugl-Meyer scale was developed as the first quantitative evaluative instrument for measuring sensorimotor stroke recovery. It is a well-designed, feasible and efficient clinical examination method that has been tested widely in the stroke population.

Robotic assisted rehabilitation also improves the direct cost-savings, and other indirect economic benefits [5]. The direct cost-savings being the decreased money spent in out of home rehabilitation, and related expenses. The indirect being the improved quality of life, and permanency of recovery.

The proposed RAD-HR was modeled based on the adult human hand articulations and range of movement. Modified Denavit-Hertenberg (DH) conventions are used in developing the kinematic model [6]. In dynamic modeling and simulation, robot parameters such as robot arm link lengths, masses, and inertia, are estimated according to the upper limb properties of a typical adult [7]. The proposed RAD-HR is supposed to be worn on the forearm and was developed to provide for the complete range of hand movements i.e., flexion/extension and radial/ulnar movements of wrist joint and fingers flexion/extension motion. Considering the dynamic modeling of human arm movement, the nonlinear computed torque control technique [6] is employed in the dynamic simulation as well as to control the proposed RAD-HR model. 
In the next section of this paper, we present an overview of the kinematic, and dynamic model for the proposed RADHR. Details on the design, and development of the proposed RAD-HR are presented in Section 3. Section 4 describes the implemented control strategy for the proposed RAD-HR, as well as the dynamics. In Section 5, experimental results are presented to evaluate the performance of the controller. Finally the paper ends with the conclusion, and the future work in Section 6.

\section{Kinematics}

The kinematic model for the RAD-HR was then based on the modified Denavit-Hartenberg (DH) method [6]. To find the modified DH parameters, we have assumed that the link frames of each successive axes of rotation coincide with the joint axes of rotation and are of the same order. In our model the joints 1, and 2 (point-A in Fig. 1) together constitute the wrist joint. Joint 1 is the flexion/extension of the wrist, and joint 2 is the radial/ulnar deviation of the wrist. Joint 3 (point-B in Fig. 1) is then the combined flexion/extension of all four fingers.

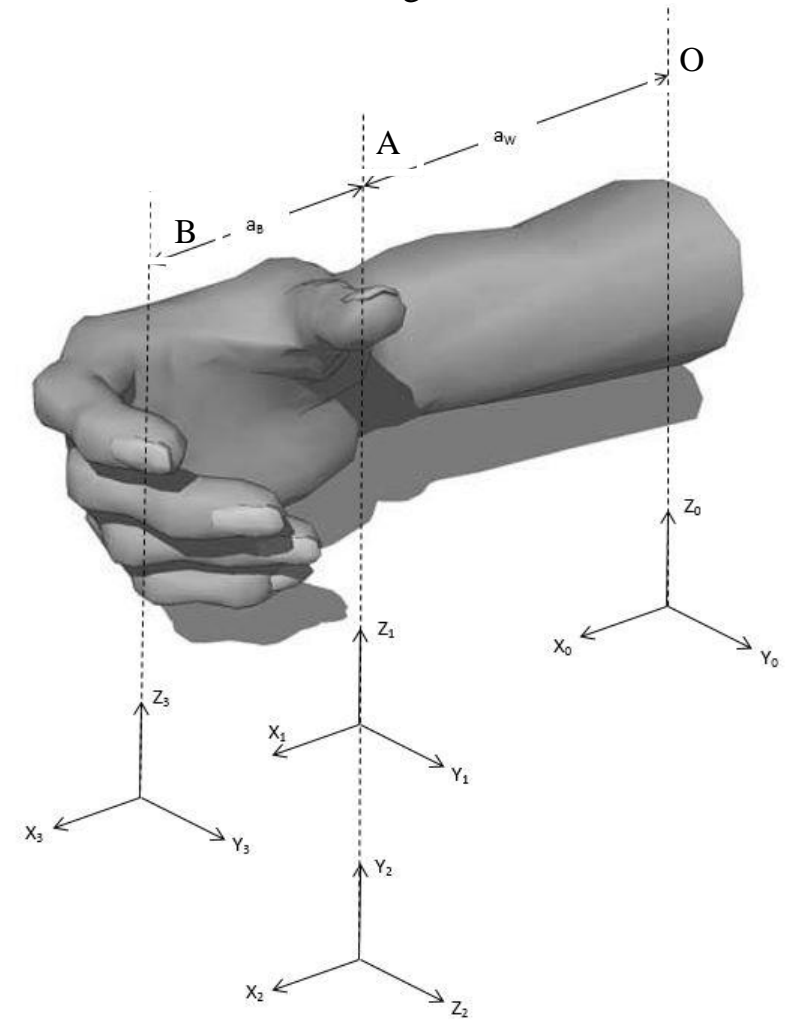

Fig. 1: Link frame assignments.

The modified DH parameters of our link frame assignments are as summarized in Table 1. These parameters are then used to represent the positions, and orientations of the frames with respect to the fixed frame of reference $\{0\}$ (point-O in Fig. 1). It is assumed that the fixed frame of reference has a distance $a_{w}$ from the reference frame $\{1\}$ (at point-A in Fig. 1).

Table 1: Modified DH parameters.

\begin{tabular}{|l|l|l|l|l|}
\hline Joint (i) & $\boldsymbol{\alpha}_{\boldsymbol{i}-\boldsymbol{1}}$ & $\boldsymbol{d}_{\boldsymbol{i}}$ & $\boldsymbol{a}_{\boldsymbol{i}-\boldsymbol{1}}$ & $\boldsymbol{\theta}_{\boldsymbol{i}}$ \\
\hline 1 & 0 & 0 & $a_{W}$ & $\theta_{1}$ \\
\hline 2 & $-\pi / 2$ & 0 & 0 & $\theta_{2}$ \\
\hline 3 & $-\pi / 2$ & 0 & $a_{B}$ & $\theta_{3}$ \\
\hline
\end{tabular}

Using the general form of a link transformation as it relates frame $\{i\}$ to the frame $\{i-1\}$. [5] 


$$
\begin{aligned}
& { }_{i}^{i-1} \mathrm{~T}=\left[\begin{array}{cc}
{ }_{i}^{i-1} R^{3 \times 3} & { }_{i}^{i-1} P^{3 \times 1} \\
0^{1 \times 3} & 1
\end{array}\right] \\
& { }_{i}^{i-1} R=\left[\begin{array}{ccc}
\cos \theta_{i} & -\sin \theta_{i} & 0 \\
\sin \theta_{i} \cos \alpha_{i-1} & \cos \theta_{i} \cos \alpha_{i-1} & -\sin a_{i-1} \\
\sin \theta_{i} \sin \alpha_{i-1} & \cos \theta_{i} \sin \alpha_{i-1} & \cos \alpha_{i-1}
\end{array}\right] \\
& { }_{i}^{i-1} P=\left[\begin{array}{lll}
a_{i-1} & -\sin \alpha_{i-1} d_{i} & \cos \alpha_{i-1} d_{i}
\end{array}\right]^{T}
\end{aligned}
$$

Where ${ }_{i}^{i-1} R$ is the rotation matrix that describes the frame $\{i\}$ in relation to the frame $\{i-1\}$. Also, ${ }_{i}^{i-1} P$ is the vector of the origin of frame $\{i\}$ relative to the frame $\{i-1\}$. The positions, and orientations of the reference frame attached to the finger joint (axis 3) with respect to the fixed reference frame $\{0\}$ can then be obtained by multiplying the individual transformation matrices.

\section{Design of the Assistive Device}

The rehabilitation capabilities of the proposed RAD-HR (Fig. 2) focuses on the basic biomechanical behaviors of an average human hand. Meaning that the focus of motion rehabilitation has been limited to the two main joints of the hand and additionally the fingers. The two main joints being where the four fingers attach to the palm/back of the hand (point-B in Fig. 1), and the wrist (point-A in Fig. 1). These joints are restricted in their ranges of motion for patient safety reasons to the specifications outlined in Table 2 . The restriction was done mostly by restricting the physical capabilities of the RAD-HR to ranges just shy of the healthy ranges of motion of the average human hand.

Table 2: Range of motion of the average human, and RAD-HR.

\begin{tabular}{|c|c|c|c|}
\hline & Motion & Average Human [8] & RAD-HR \\
\hline \multirow{3}{*}{ Wrist } & Radial Deviation & $20^{\circ}$ & $22^{\circ}$ \\
\cline { 2 - 4 } & Ulnar Deviation & $30^{\circ}$ & $22^{\circ}$ \\
\cline { 2 - 4 } & Flexion & $70^{\circ}$ & $90^{\circ}$ \\
\cline { 2 - 4 } & Extension & $90^{\circ}$ & $90^{\circ}$ \\
\hline \multirow{2}{*}{ Fingers } & Flexion/Extension & $90^{\circ}$ & $90^{\circ}$ \\
\hline
\end{tabular}

The device is attached to the patients forearm, palm, thumb, and fingers. Using three micro linear electric servos to help rotate the hand. The actuator situated on the back of the hand operates the flexion/extension of the fingers. This flexion/extension manipulates a separate sub-mechanism attached to the thumb. As the fingers go into flexion the thumb will also, and vice versa. The two servos mounted on top of the forearm aid in the flexion/extension (both extend fully or retract to half extension), and radial/ulnar deviation (one extends fully one retracts fully) of the wrist.

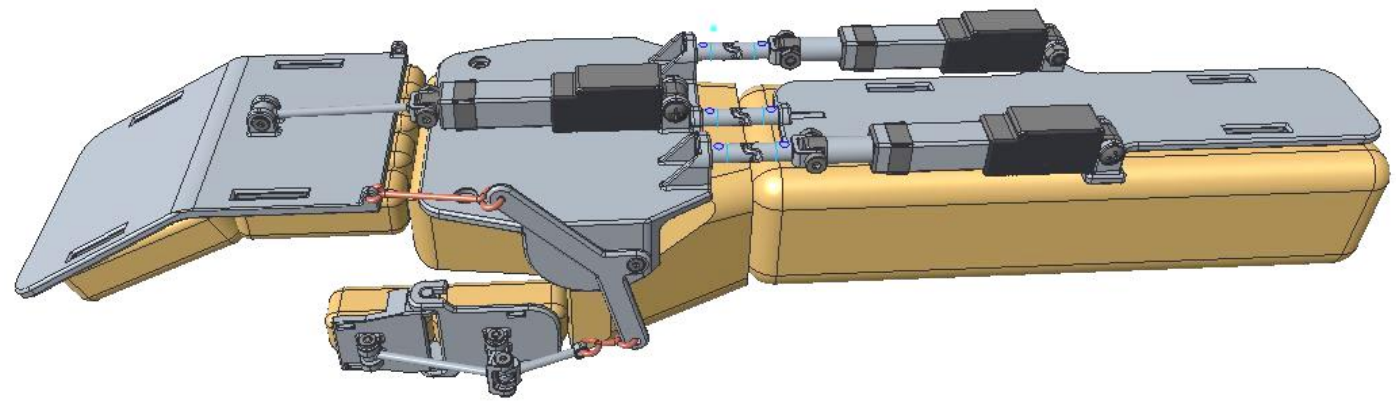

Fig. 2: CAD model of robotic assistive device for hand rehabilitation (RAD-HR).

\section{Control}

The dynamic behaviour of the proposed RAD-HR can be expressed by the rigid body dynamic equation as: 


$$
\tau=M(\theta) \ddot{\theta}+V(\theta, \dot{\theta})+G(\theta)
$$

where, $\theta \in \mathbb{R}^{3}$ is the joint variables vector, $\tau \in \mathbb{R}^{3}$ is the generalized torques vector, $M \in \mathbb{R}^{3 \times 3}$ is the inertia matrix, $V(\theta, \dot{\theta}) \in \mathbb{R}^{3}$ is the coriolis/centrifugal vector, and $G(\theta) \in \mathbb{R}^{3}$ is the gravity vector. Equation (4) can be written as:

$$
\ddot{\theta}=-M^{-1}(\theta)[V(\theta, \dot{\theta})+G(\theta)]+M^{-1}(\theta) \tau
$$

$M^{-1}(\theta)$ always exists since $M(\theta)$ is symmetrical and positive definite [9].

To properly manage, direct, and regulate the behaviour of the proposed RAD-HR we have employed nonlinear computed torque control (CTC) technique. Schematic diagram of the CTC is shown in Fig. 3.

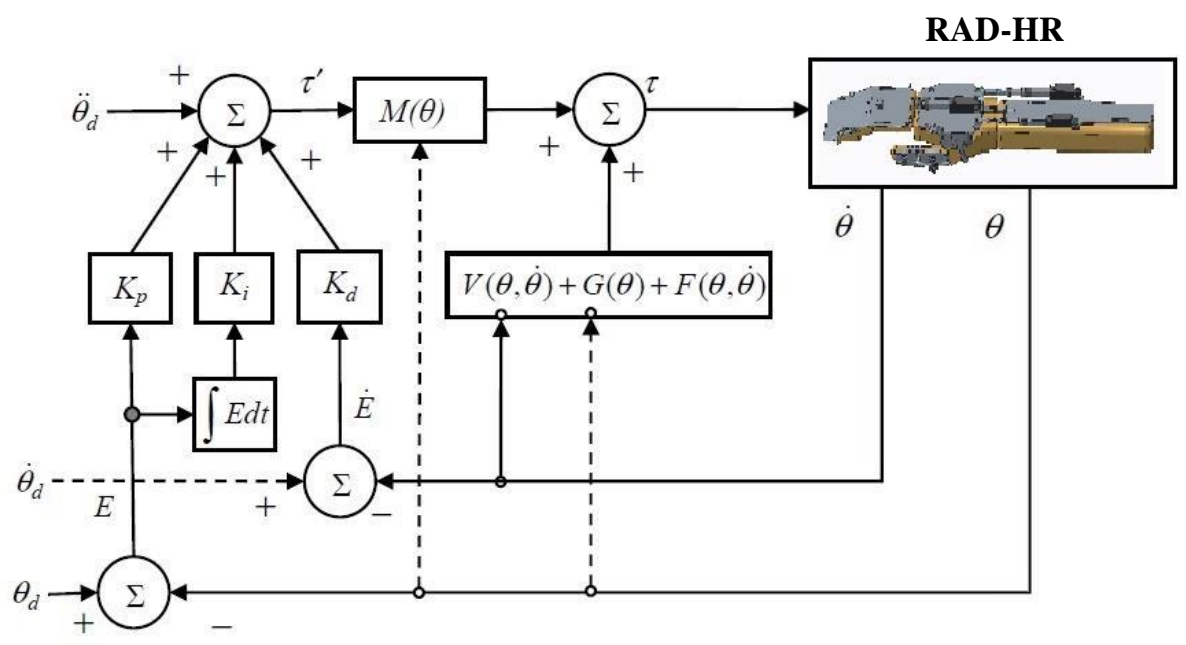

Fig. 3: Schematic diagram of computed torque control.

An integral (of error) term was added to the controller to improve the tracking performance, and to compensate for the trajectory tracking error that occurs due to imperfect dynamic modelling, and parameter estimation. The variables $K_{p}, K_{d}$, and $K_{i}$ are positive definite diagonal matrices defining the proportional, derivative, and integral gain, respectively. We find the error vector $E$, and its derivatives are as:

$$
\begin{gathered}
E=\theta_{d}-\theta ; \dot{E}=\dot{\theta}_{d}-\dot{\theta} ; \ddot{E}=\ddot{\theta}_{d}-\ddot{\theta} \\
\ddot{E}+K_{d} \dot{E}+K_{p} E+K_{i} \int E d t=0
\end{gathered}
$$

This allows us to fine tune the control gains $\left(K_{p}, K_{d}\right.$, and $\left.K_{i}\right)$ giving us a greatly improved the stability, and accuracy of our system.

\section{Simulation}

Simulations were carried out to test the accuracy of the proposed control system. These simulations follow preprogrammed trajectories corresponding to traditional passive rehabilitation methods [10]. The simulation run was of the three joints moving individually. The first exercise is the radial/ulnar deviation of the hand, or the movement of bending the wrist to and away from the thumb. Next is the flexion/extension of the wrist, or the movement of the palm towards and away from the wrist. Lastly is the flexion/extension of the fingers, which is the movement of the fingers toward and away from the palm. The simulations were conducted with a reference trajectory (red dotted line) or the desired motion, and the measured (solid blue line) which would be the outcome of the controller emulation (Fig. 4 row A). This allows us to measure the error for each joint. Due to very low error $\left(<0.5^{\circ}\right.$, Fig. 4 row B) the two lines are nearly indistinguishable. The error is a function of 
time which is the deference between reference and measured trajectories. This shows us that the maximum tracking error was less than $0.5^{\circ}$.
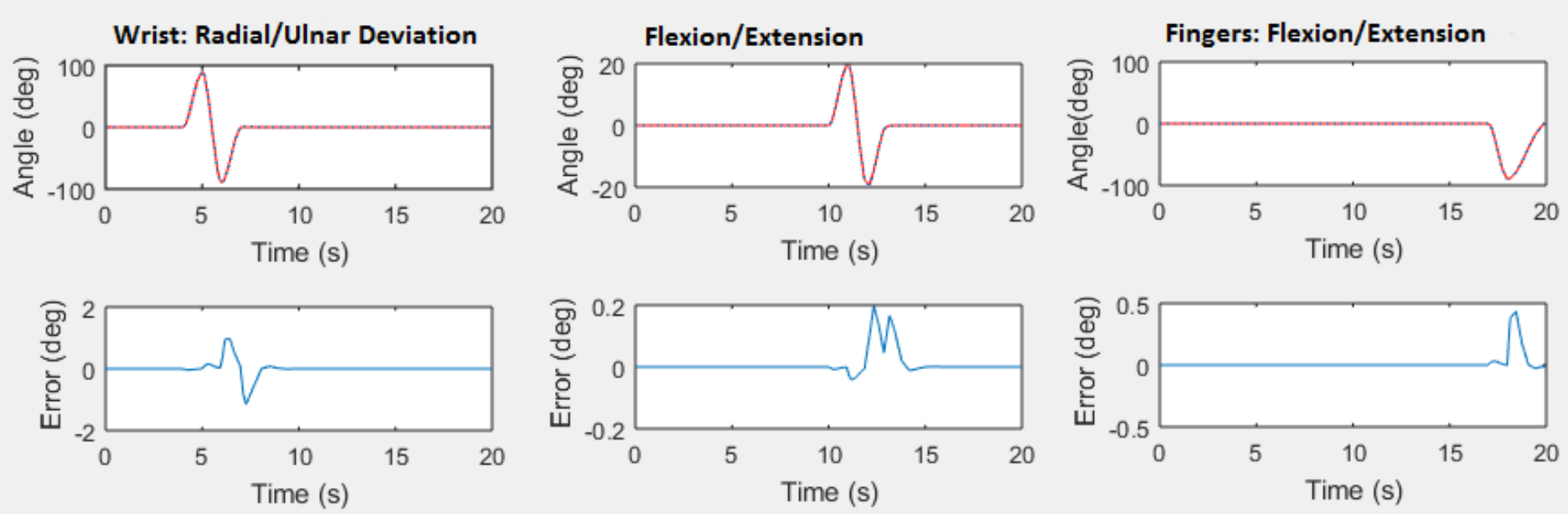

Fig. 5: Simulated passive rehabilitation exercise of the wrist in radial/ulnar deviation, flexion/extension, and fingers in flexion/extension.

\section{Conclusion}

The kinematic, and dynamic model of the RAD-HR which corresponds to the average movement of a human hand is presented. The nonlinear computed torque control technique was used in the dynamic simulation. Where the trajectory tracking corresponds to typical passive rehabilitation exercises.

Current work aims to develop the proposed RAD-HR based on its kinematic model. Our future work seeks to introduce an electromyogram based controller to aid in the rehabilitation and use of the RAD-HR.

\section{Acknowledgements}

The first author gratefully acknowledges the support provided for this research through McNair and SURF scholarships.

\section{References}

[1] A. S. Go, D. Mozaffarian, V. L. Roger, E. J. Benjamin, J. D. Berry, M. J. Blaha, S. Dai, E. S. Ford, C. S. Fox, S. Franco, and H. J. Fullerton, "Heart disease and stroke statistics--2014 update: a report from the American Heart Association," Circulation, vol. 129, no. 3, pp. e28, 2014.

[2] S. Masiero, A. Celia, G. Rosati, and M. Armani, "Robotic-assisted rehabilitation of the upper limb after acute stroke," Archives of physical medicine and rehabilitation, vol. 88, no. 2, pp. 142-9, 2007.

[3] M. H. Rahman, M. Saad, J. P. Kenné, and P. S Archambault, "Exoskeleton Robot for Rehabilitation of Elbow and Forearm Movements," in IEEE The 18th Mediterranean Conference on Control and Automation, MED'10, pp. 1562$1572,2010$.

[4] P. S. Lum, C.G. Burgar, P. C. Shor, M. Majmundar, and M. Van der Loos, "Robot-Assisted Movement Training Compared with Conventional Therapy Techniques for the Rehabilitation of Upper-Limb Motor Function After Stroke," Archives of Physical Medicine and Rehabilitation, vol. 83, no. 7, pp. 952-959, 2002.

[5] G. R. Romer, H. J. Stuyt, and A. Peters, "Cost-savings and economic benefits due to the assistive robotic manipulator (ARM)," in 9th International Conference on Rehabilitation Robotics, ICORR, 2005, pp. 201-204.

[6] J. J. Craig, Introduction to Robotics Mechanics and Control, 3rd Ed. Pearson Prentice Hall, 2004.

[7] A. D. Winter, Biomechanics and Motor Control of Human Movements, $2^{\text {nd }}$ Ed. Canada: University of Waterloo Press, 1992.

[8] N. P. Hamilton, Kinesiology: Scientific basis of human motion. Brown \& Benchmark, 2011. 
[9] M. H. Rahman, T. Kittel-Ouimet, and M. Saad, "Dynamic Modeling and Evaluation of a Robotic Exoskeleton for Upper-Limb Rehabilitation," International Journal of Information Acquisition, vol. 8, no. 1, pp. 83-102, 2011.

[10] D. J. Magee, J. E. Zachazewski, and W. S. Quillen, Pathology and intervention in musculoskeletal rehabilitation. Elsevier Health Sciences, 2008. 